\title{
Automatic 3D Segmentation of Intravascular Ultrasound Images Using Region and Contour Information
}

\author{
Marie-Hélène Roy Cardinal ${ }^{1}$, Jean Meunier ${ }^{2}$, Gilles Soulez ${ }^{3}$, Roch L. Maurice ${ }^{1}$, \\ Éric Thérasse ${ }^{3}$, and Guy Cloutier ${ }^{1}$ \\ 1 Laboratory of Biorheology and Medical Ultrasonics, \\ University of Montreal Hospital, Canada \\ roycarmh@iro.umontreal.ca, guy.cloutier@umontreal.ca \\ 2 Département d'Informatique et de Recherche Opérationnelle, \\ University of Montreal, Canada \\ 3 Radiology Department, University of Montreal Hospital, Canada
}

\begin{abstract}
Intravascular ultrasound (IVUS) produces images of arteries that show the lumen in addition to the layered structure of the vessel wall. A new automatic 3D IVUS fast-marching segmentation model is presented. The method is based on a combination of region and contour information, namely the gray level probability density functions (PDFs) of the vessel structures and the image gradient. Accurate results were obtained on in-vivo and simulated data with average point to point distances between detected vessel wall boundaries and validation contours below $0.105 \mathrm{~mm}$. Moreover, Hausdorff distances (that represent the worst point to point variations) resulted in values below $0.344 \mathrm{~mm}$, which demonstrate the potential of combining region and contour information in a fast-marching scheme for 3D automatic IVUS image processing.
\end{abstract}

\section{Introduction}

Intravascular ultrasound (IVUS) is a medical imaging technique that produces cross-sectional images of the vascular wall as a catheter is pulled-back inside blood vessels. The ability of IVUS to characterize the vascular wall was initially proven in 1989 [1]. Since then, it has become very useful for studying atherosclerotic disease because quantitative assessment of the wall, information about the nature of atherosclerotic lesions as well as plaque shape and size are provided. IVUS is expected to play an important role in atherosclerosis research; for example, to achieve precise evaluation of the disease in progression-regression therapies [2. Experts agree that IVUS imaging adds precious complementary information to angiography which only shows a projection of the lumen [3].

A typical intravascular ultrasound acquisition contains several hundred of images making non-automatic analysis of the data long, fastidious and subject to high intra- and inter- observer variabilities. These could be serious constraints against the clinical usage of IVUS. There is also some quality issues related to IVUS imaging due to speckle noise, imaging artifacts and shadowing of parts of 
the vessel wall by calcifications. It is thus necessary to develop specific segmentation methods that take into account the nature of IVUS images.

So far, a number of segmentation techniques have been developed for IVUS data analysis. A great portion of this work was based on local properties of image pixels, namely gradient-based active surfaces [4] or multi-agents [5] and pixel intensity combined to gradient active contours [6]. Graph search was also investigated using different edge operators [7, 8, 9. Another portion of the IVUS segmentation work was based on more global or region information. Texturebased morphological processing was considered [10. Gray level variances were then used for the optimization of a maximum a posteriori (MAP) estimator modeling ultrasound speckle and contour geometry 11. However, some of these methods are semi-automatic and others focus on either region or contour information without combining them.

The aim of this work was thus to achieve automatic IVUS image analysis by combining region and contour features in a segmentation model. Since pixel gray values are distributed according to Rayleigh probability density functions (PDFs) in B-mode imaging of uniform scattering tissues 12, PDF features can be of value for IVUS segmentation. In a previous semi-automatic $2 \mathrm{D}$ segmentation model 13], gradient- and PDFs-based methods were compared and both provided results with similar variability (PDFs parameters were calculated from manually traced regions). The combination of the region statistics and of the gray level gradient contour information, that are complementary, is thus hypothetically more suitable for IVUS image analysis, especially when the vascular wall edges are weakly defined. These features were included in a multiple interface fast-marching segmentation [14], that enables simultaneous segmentation of different parts of the vessel wall. In this paper, automatic estimation of the PDF parameters and initial contours is also presented.

\section{Methods}

\subsection{Fast-Marching Segmentation}

The fast-marching method was derived from the level-set model; they both allow the following of an interface propagating under a speed function $F$ [15]. Fastmarching can be applied to image segmentation by interpreting an image contour as the propagating interface final position [16. To stop the evolution of the interface on image boundaries, the speed function, that is defined in terms of image or shape features, should become close to zero when the propagating front meets with object boundaries.

In the fast-marching formulation, where an interface propagates under a unidirectional speed function, the evolution of the contour is expressed in terms of the arrival time $T(x)$ of the front at point $x=\left(x_{1}, x_{2}, \ldots, x_{n}\right) \in \Re^{n}$. The $T$ function satisfies Eq. 1], stating that the arrival time difference between two adjacent pixels increases as the velocity of the contour decreases.

$$
|\nabla T| F=1 .
$$


The propagation of the interface is done in $3 \mathrm{D}$ via the construction of the arrival time function map ( $T$ map). The construction algorithm [17] selects the interface point having the smallest arrival time and calculates the arrival times of its neighbors. Neighbor arrival times are updated by solving a discrete 3D approximation of Eq. 1. This is repeated until the interface has propagated across the whole image or until the interface front is considered stationary (when the time gradient is sufficiently high).

In IVUS imaging, multiple contours (lumen and media) must be identified to characterize plaque development, consequently segmentation was done via a multiple interface extension of the fast-marching algorithm [14]. For this particular case, a boundary is defined by the meeting position of two contours propagating in opposite directions. A speed function must then be determined for each propagating interface; it is defined according to the PDF of the anatomical structure in which the interface is evolving. The $T$ map is built by selecting the point with the smallest arrival time value from all interfaces 1 . For the current study, the multiple interfaces evolved according to the speed function given by Eq. 2, which is based on the gray values occurring probability and gradient.

$$
\begin{gathered}
F_{m}(i, j, k)=\frac{1}{N_{\nu}} \sum_{s \in \nu} p_{m}\left(I_{s}\right)+1 /\left(1+g\left(I_{s}\right)\right) . \\
g=\left|\nabla G_{\sigma} * I\right|
\end{gathered}
$$

where $I_{s}$ is the gray level value of pixel $s$ in image $I ; \nu$ is the set of the $N_{\nu}$ neighbors of the pixel positioned at $(i, j, k)$ (the neighbors window size is $7 \times 7 \times 3$ ); $p_{m}\left(I_{s}\right)$ is the occurring probability of pixel $I_{s}$ in the region $m ; g\left(I_{s}\right)$ is the value of the gradient $g$ at pixel $I_{s}$ and $G_{\sigma}$ is a gaussian smoothing filter of standard deviation $\sigma=2$. According to the first term of Eq. 2 the velocity of interface $m$ will take higher values when it is inside a region having a grayscale distribution close to $p_{m}$; it will decrease when approaching the boundary since the neighbors are distributed under other component PDFs.

\section{$2.2 \quad$ Pre-processing}

The pre-processing calculations involved the catheter artifact detection and removal; and the feature extraction (PDF mixture parameters and image gradient).

The pixels near the catheter with gray level values that correlated through the whole IVUS pullback were labeled as being part of the ring-down artifact. The catheter artifact was removed from the images by subtracting the average ring-down gray level values from each IVUS 2D frame. The catheter artifact had to be detected before each segmentation because it is specific to each pullback.

The speed function of Eq. 2 uses the gray level distributions $p_{m}\left(I_{s}\right)$ of the different vessel wall structures. The distribution of a whole IVUS pullback was thus estimated by a mixture of Rayleigh PDFs, each corresponding to the lumen, plaque structure of the intima, media, and adventitia plus surrounding tissues. The mixture $\operatorname{PDF} p_{X \mid \Theta}$ with parameters $\Theta=\left\{\left(\omega_{m}, a_{m}^{2}\right)\right\}_{m=1}^{M}$ is:

\footnotetext{
${ }^{1}$ A detailed description of the $T$ map construction algorithm can be found in 14 .
} 


$$
\begin{aligned}
p_{X \mid \Theta}(x \mid \Theta) & =\sum_{m=1}^{M} \omega_{m} p_{m}\left(x \mid a_{m}^{2}\right) . \\
p_{m}\left(x \mid a_{m}^{2}\right) & =\frac{x}{a_{m}^{2}} \exp \left(-\frac{x^{2}}{2 a_{m}^{2}}\right)
\end{aligned}
$$

where $X$ represents the pixels' gray level taking values in $[0, \ldots, 255]$ (with $x>0$ ); $M$ is the number of different tissue structures; $\omega_{m}$ is the proportion of the $m^{\text {th }}$ component of the mixture; and $p_{m}\left(x \mid a_{m}^{2}\right)$ is the $m^{\text {th }}$ tissue Rayleigh probability density function with parameter $a_{m}^{2}$, which is related to the variance $\sigma_{m}^{2}=a_{m}^{2}(4-\pi) / 2$ of the distribution.

The mixture parameters $\Theta$ were estimated with the ExpectationMaximization algorithm (EM) that is an iterative computation technique of maximum likelihood estimates for incomplete data [18].

\subsection{Automatic Initialization}

Initial contours must be computed to initialize the fast-marching segmentation. These contours were found on one every three frames to diminish computational load without losing their 3D continuity.

An initial lumen contour was first automatically detected from the IVUS data. The log-likelihood of the lumen $L_{\text {lum }}$ was calculated for each pixel according to Eq. 6 and by using the previously estimated lumen distribution of the PDF mixture.

$$
L_{\text {lum }}(i, j, k)=\frac{1}{N_{\nu}} \sum_{s \in \nu} \log p_{\text {lum }}\left(I_{s}\right) .
$$

where $p_{\text {lum }}\left(I_{s}\right)$ is the estimated occurring probability of pixel $I_{s}$ in the lumen region.

The initial lumen contour was set as a smooth closed curve, not necessarily circular to preserve the irregular lumen shape, containing the catheter and the pixels maximizing $L_{\text {lum }}$. The initial media contour calculation was performed similarly to the lumen initialization. An elliptical contour surrounding the previously found lumen was grown radially to maximize the media log-likelihood $L_{m e d}$ also calculated according to Eq. 6] but with the estimated media distribution $p_{\text {med }}\left(I_{s}\right)$. An elliptical contour was searched because the media boundary is generally smooth and regular (it appears as a hypoechoic ring, see Fig. 2).

These automatically computed initial contours were only rough estimates of the wall boundaries; they were used to initialize the fast-marching segmentation that allowed obtaining more precise contours. The initial contour points were spline-interpolated axially and longitudinally while being forbidden to overlap to preserve the layered structure of the vessel wall. The fast-marching requires the initial front to be inside the object to segment, the contours were thus each shifted radially (10 pixels) in the 2 opposite directions to compute the 2 pairs of $3 \mathrm{D}$ tube-like initial interfaces. The interfaces propagating in opposite directions (and including the exact object border) were thus separated by 20 pixels. 


\subsection{In-Vivo Data and Numerical Simulation}

An in-vivo IVUS pullback of 600 frames from a diseased superficial femoral artery was acquired with a Volcano Therapeutics equipment (In-vision gold, California, USA) using a $20 \mathrm{MHz}$ transducer. Images of size $10 \times 10 \mathrm{~mm}$ were digitized on 384 x 384 pixel matrices and stored using the DICOM standard. The acquisition was done at a 10 images/sec frame rate and the catheter pullback velocity was set to $1 \mathrm{~mm} / \mathrm{sec}$ generating $0.1 \mathrm{~mm}$ thick $2 \mathrm{D}$ slices.

In addition to the in-vivo data, realistic simulations of IVUS data were conducted. Since the exact geometry of simulated data is known, direct calculation of the detected boundary performance could be obtained. A detailed description of the image-formation model used to simulate IVUS echograms can be found in [19]. To generate a realistic vessel geometry, the vessel boundaries (lumen, plaque of the intima, media) were created from manually traced contours on an in-vivo IVUS series. The simulated pullback contained 86 IVUS 2D frames.

The automatic 3D segmentation method was applied to the in-vivo and simulated IVUS images. For validation purpose, the in-vivo IVUS segmentation results were compared with manually traced contours on 1 every 10 frames; boundary position from 60 IVUS frames were thus available. Results on simulated data were compared with the exact geometry. The average and Hausdorff point to point contour distances [20, and wall layer area differences were calculated. Hausdorff distance represents the worst case, it is the maximum distance between between corresponding contour points.

\section{Results}

As part of the pre-processing, the EM algorithm was applied to the IVUS series to evaluate the PDF mixture parameters. An example of an automatically detected Rayleigh PDF mixture and corresponding IVUS pullback histogram is shown in Fig. [1].

Segmentation results for the automatic 3D fast-marching method combining PDFs and gradient are shown in Fig. 2, The lumen and media detected boundaries are presented for typical cross-sectional IVUS images of the whole 3D in-vivo and simulated pullbacks. Table 1 shows the average and Hausdorff

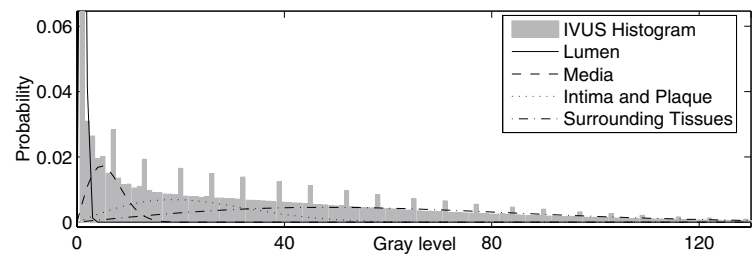

Fig. 1. Illustration of an automatically detected Rayleigh PDF mixture and corresponding IVUS gray level histogram 


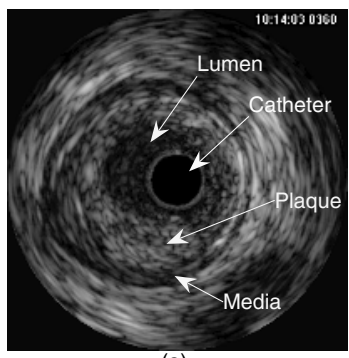

(a)

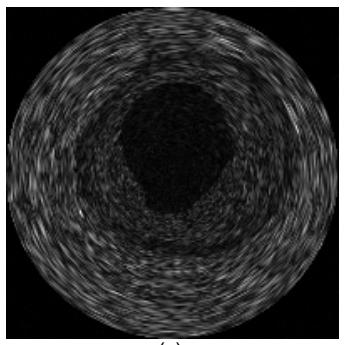

(c)

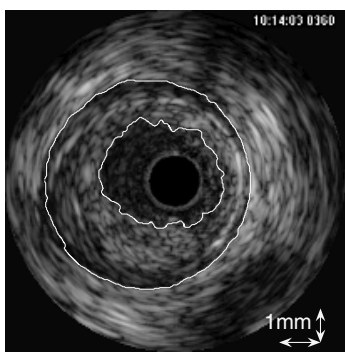

(b)

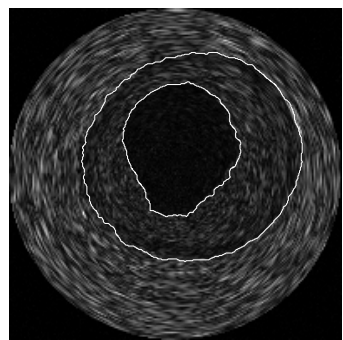

(d)

Fig. 2. Segmentation examples on in-vivo and simulated data. Typical IVUS crosssectional image (a). Lumen and media detected contours (b). Simulated IVUS crosssectional image (c). Lumen and media detected contours (d).

Table 1. Average distances, Hausdorff distances and area differences between detected contours on in-vivo and simulated data and the validation contours

\begin{tabular}{lll}
\hline \multicolumn{3}{c}{ In-vivo Data } \\
\hline \multicolumn{1}{c}{ Lumen } & \multicolumn{1}{c}{ Media } \\
\hline \hline Average Distances $(\mathrm{mm})$ & $0.083 \pm 0.111$ & $0.105 \pm 0.094$ \\
Hausdorff Distances $(\mathrm{mm})$ & $0.344 \pm 0.237$ & $0.300 \pm 0.104$ \\
Area Differences $\left(\mathrm{mm}^{2}\right)$ & $-0.24 \pm 1.07(\mathrm{r}=0.974)$ & $-0.47 \pm 1.14(\mathrm{r}=0.890)$ \\
\hline & Simulated Data \\
\hline \hline Average Distances $(\mathrm{mm})$ & $0.059 \pm 0.069$ & $0.066 \pm 0.061$ \\
Hausdorff Distances $(\mathrm{mm})$ & $0.235 \pm 0.105$ & $0.224 \pm 0.064$ \\
Area Differences $\left(\mathrm{mm}^{2}\right)$ & $0.18 \pm 0.65(\mathrm{r}=0.997)$ & $-0.65 \pm 0.73(\mathrm{r}=0.976)$ \\
\hline
\end{tabular}

The pixel size is $26 \times 26 \mu \mathrm{m}^{2}$.

distances and the area differences between the detected contours and the validation boundaries for the in-vivo and simulated IVUS pullbacks.

\section{Discussion}

The goal of this work was to demonstrate the feasibility of a fully automatic 3D fast-marching method for the segmentation of IVUS images when using PDFs and gradient in the same model. 
No ground truth value was available to evaluate the precision of the calculated mixture parameters, however a rough comparison, in Fig. 1, of the pullback gray level histogram with the estimated Rayleigh PDF mixture showed good agreement. A qualitative analysis of the automatic fast-marching segmentation combining region and contour information in Fig. 2 revealed detected contours that were very close to all vessel layers. Fig. 2 also showed that noncircular lumen contours could be precisely found. For the simulated images, Table 1 showed that the detected contours were very close to the simulated vessel boundaries with average point to point distances below $0.066 \mathrm{~mm}$ ( 2.5 pixels). Similar distances were obtained for the in-vivo IVUS data with average distances between 0.083 and $0.105 \mathrm{~mm}$ ( 3 to 4 pixels). Hausdorff distances, which represent the highest distance between the contour and the validation boundary position, resulted in values below $0.344 \mathrm{~mm}$ (13 pixels), this demonstrates the efficiency of the segmentation method. Area differences between detected and validation contours showed in Table 1 were slightly higher than the ones reported in [5,8] (between $-0.14 \pm 1.01$ and $0.27 \pm 0.49 \mathrm{~mm}^{2}$ ), but the data was acquired on a femoral artery that is larger than the coronary arteries of these studies.

These preliminary segmentation results showed that this new IVUS segmentation approach is promising for the processing of large pullbacks without user interaction. In the near future, the segmentation method will be applied to several IVUS series acquired from different patients before and after undergoing balloon angioplasty to characterize the lumen and wall volumic changes over a whole vessel segment. The complete validation of the segmentation method will also include several experts' manually traced contours repeated in time to assess the performance in terms of intra- and inter-user variabilities.

Acknowledgments. This work was supported by grants from the Canadian Institute of Health Research (G.C., G.S., \#MOP-53244) and Valorisation-Recherche Québec (group grant \#2200-094), and by a National Scientist award (G.C.), a clinical research scholarship award (G.S.) and a studentship award (M.H.R.C.) from the Fonds de la Recherche en Santé du Québec. We also acknowledge the work of Zhao Qin.

\section{References}

1. Gussenhoven, E.J., Essed, C.E., Lancée, C.T., Mastik, F., Frietman, P., van Egmond, F.C., Reiber, J., Bosch, H., van Urk, H., Roelandt, J., Bom, N.: Arterial wall characteristics determined by intravascular ultrasound imaging: An in vitro study. J. Am. Coll. Cardiol. 14 (1989) 947-952

2. Nissen, S.E.: Application of intravascular ultrasound to characterize coronary artery disease and assess the progression or regression of atherosclerosis. Am. J. Cardiol. 89 (2002) 24B-31B

3. Nissen, S.E., Yock, P.: Intravascular ultrasound: Novel pathophysiological insights and current clinical applications. Circulation 103 (2001) 604-616 
4. Klingensmith, J.D., Shekhar, R., Vince, D.G.: Evaluation of three-dimensional segmentation algorithms for the identification of luminal and medial-adventitial borders in intravascular ultrasound images. IEEE Trans. Med. Imag. 19 (2000) 996-1011

5. Bovenkamp, E.G.P., Dijkstra, J., Bosch, J.G., Reiber, J.H.C.: Multi-agent segmentation of IVUS images. Pattern Recogn. 37 (2004) 647-663

6. Kovalski, G., Beyar, R., Shofti, R., Azhari, H.: Three-dimensional automatic quantitative analysis of intravascular ultrasound images. Ultrasound Med. Biol. 26 (2000) 527-537

7. Zhang, X., McKay, C.R., Sonka, M.: Tissue characterization in intravascular ultrasound images. IEEE Trans. Med. Imag. 17 (1998) 889-899

8. Koning, G., Dijkstra, J., von Birgelen, C., Tuinenburg, J.C., Brunette, J., Tardif, J.C., Oemrawsingh, P.W., Sieling, C., Melsa, S., Reiber, J.H.C.: Advanced contour detection for three-dimensional intracoronary ultrasound: A validation - in vitro and in vivo. Int. J. Cardiac Imag. 18 (2002) 235-248

9. von Birgelen, C., Mario, C.D., Li, W., Schuurbiers, J.C.H., Slager, C.J., de Feyter, P.J., Serruys, P.W., Roelandt, J.R.T.C.: Morphometric analysis in threedimensional intracoronary ultrasound : An in vitro and in vivo study using a novel system for the contour detection of lumen and plaque. Am. Heart J. 132 (1996) $516-527$

10. Mojsilovic, A., Popovic, M., Amodaj, N., Babic, R., Ostojic, M.: Automatic segmentation of intravascular ultrasound images: A texture-based approach. Ann. Biomed. Eng. 25 (1997) 1059-1071

11. Haas, C., Ermert, H., Holt, S., Grewe, P., Machraoui, A., Barmeyer, J.: Segmentation of 3D intravascular ultrasonic images based on a random field model. Ultrasound Med. Biol. 26 (2000) 297-306

12. Wagner, R.F., Smith, S.W., Sandrik, J.M., Lopez, H.: Statistics of speckle in ultrasound B-scans. IEEE Trans. Son. Ultrason. 30 (1983) 156-163

13. Roy Cardinal, M.H., Meunier, J., Soulez, G., Thérasse, E., Cloutier, G.: Intravascular ultrasound image segmentation : A fast-marching method. In: Lecture Notes in Computer Sciences. Proccedings of MICCAI 2003. Volume 2879. (2003) 432-439

14. Sifakis, E., Garcia, C., Tziritas, G.: Bayesian level sets for image segmentation. J. Vis. Commun. Image R. 13 (2002) 44-64

15. Osher, S., Sethian, J.A.: Fronts propagating with curvature-dependent speed: Algorithms based on hamilton-jacobi formulations. J. Comput. Phys. 79 (1988) 12-49

16. Malladi, R., Sethian, J.A., Vemuri, B.C.: Shape modeling with front propagation: A level set approach. IEEE Trans. Pattern Anal. Machine Intell. 17 (1995) 158-175

17. Sethian, J.A.: A fast marching level set method for monotonically advancing fronts. Proc. Nat. Acad. Sci. 93 (1996) 1591-1595

18. Dempster, A.P., Laird, N.M., Rubin, D.B.: Maximum likelihood from incomplete data via the EM algorithm. J. Roy. Stat. Soc. B 39 (1977) 1-38

19. Maurice, R.L., Ohayon, J., Finet, G., Cloutier, G.: Adaptating the Lagrangian speckle model estimator for endovascular elastography: Theory and validation with simulated radio-frequency data. J. Acoust. Soc. Am. 116 (2004) 1276-1286

20. Chalana, V., Kim, Y.: A methodology for evaluation of boundary detection algorithms on medical images. IEEE Trans. Med. Imag. 16 (1997) 642-652 